\title{
How Well Can Ants Color Graphs?
}

\author{
Aleksander Vesel ${ }^{1}$ and Janez Žerovnik ${ }^{2}$ \\ ${ }^{1}$ Department of Mathematics, PEF, University of Maribor, Maribor, Slovenia \\ 2 Faculty of Mechanical Engineering, University of Maribor, Maribor, Slovenia and Department of Theoretical Computer \\ Science, IMFM, Ljubljana, Slovenia
}

\begin{abstract}
We compare the ants algorithm for graph coloring recently proposed by Costa and Hertz with the repeated Recursive Largest First algorithm and with a PetfordWelsh type algorithm. In our experiments, the latter is much better than the first two.
\end{abstract}

Keywords: graph coloring, ants algorithm, PetfordWelsh, RLF.

\section{Introduction}

Combinatorial optimization problems arise in situations where discrete choices must be made and solving them amounts to finding an optimal solution among a finite or countably infinite number of alternatives. Optimality relates to some cost criterion, which provides a quantitative measure of the quality of each solution. This area of discrete mathematics is of great practical use and has attracted much attention.

Many combinatorial optimization problems are NP-hard (see, for example [8], a classical introduction to the theory of computational complexity). It is generally believed that NP-hard problems cannot be solved to optimality within times which are polynomially bounded functions of input size. Therefore, there is much interest in approximation algorithms which can find near-optimal solutions within reasonable running times.

One of the most studied NP-hard problems is the graph coloring problem [10]. Graph coloring has numerous applications in scheduling [9] and other practical problems including register allocation, the design and operation of flexible manufacturing systems [16], frequency assignment (see [3] and references there) etc. It is well-known that the problem of determining the chromatic number is NP-hard on arbitrary graphs. Costa and Hertz [6] recently proposed an evolutionary algorithm which imitates the behaviour of ants for solving the graph coloring problem. The evolutionary methods use the collective properties of a group of distinguishable solutions, called a population.

There are many other approaches to graph coloring [10]. In this paper, we have chosen two in order to compare them with the ants algorithm.

The ants algorithm proposed in [6] uses the algorithm called Recursive Largest First (RLF) as a subroutine. Therefore, it is natural to compare the ants algorithm with a simple repetition of independent RLF runs within the same time bounds. The algorithm RLF belongs to the class of constructive methods. They build feasible solutions by starting from an empty solution and by repeatedly inserting a new component into the current partial solution.

The algorithm antivoter was proposed by Petford and Welsh [12]. It is a randomized algorithm based on an analogy to a model of infinite particle systems from statistical mechanics. It can be shown that the algorithm is indeed a metropolis type algorithm and is also equivalent to a generalized Boltzmann machine neural network [13]. In this paper we use an extension of the Petford-Welsh algorithm $[19,3]$.

'Ants can color graphs' was the message of the paper [6]. However, experimental results in the cited paper only compare various variants of the ants algorithm. A comparison with RLF is not realistic, because the ants algorithm was run for an order of magnitude longer times. On a basis of such an experiment one cannot conclude 


\begin{tabular}{|r|r||l||l|l||l|l|l|}
\hline \multicolumn{1}{|l||}{} & \multicolumn{1}{c||}{ RLF } & \multicolumn{2}{c||}{ ANTCOL } & \multicolumn{3}{c|}{ Petford-Welsh } \\
$n$ & $p$ & & RLF & DSATUR & $T=0.2$ & $T=0.3$ & $T=0.4$ \\
\hline \multirow{3}{*}{100} & 0.4 & 13.10 & 12.65 & 12.80 & 12.50 & 12.05 & 12.10 \\
& 0.5 & 16.05 & 15.40 & 15.65 & 15.25 & 14.95 & 14.95 \\
& 0.6 & 19.85 & 18.65 & 18.85 & 18.35 & 18.00 & 18.35 \\
\hline \multirow{3}{*}{300} & 0.4 & 30.25 & 29.20 & 36.00 & 27.95 & 26.90 & 28.15 \\
& 0.5 & 38.45 & 36.30 & 44.90 & 34.80 & 33.40 & 36.05 \\
& 0.6 & 47.50 & 44.40 & 55.00 & 42.55 & 41.45 & 45.55 \\
\hline \multirow{3}{*}{500} & 0.4 & 46.00 & 46.40 & 54.00 & 42.00 & 40.25 & 44.05 \\
& 0.5 & 58.60 & 56.00 & 68.20 & 52.60 & 50.70 & 56.70 \\
& 0.6 & 73.00 & 68.80 & 84.20 & 64.80 & 63.90 & 71.35 \\
\hline \multirow{3}{*}{1000} & 0.4 & 82.10 & 91.50 & 95.50 & 74.20 & 71.50 & 81.50 \\
& 0.5 & 105.05 & 111.00 & 121.00 & 94.15 & 93.00 & 105.15 \\
& 0.6 & 132.00 & 127.50 & 151.00 & 116.40 & 118.60 & 132.30 \\
\hline
\end{tabular}

Table 1. Comparison of short runs (nants $=100,5000 \mathrm{RLF}, 5000 n \mathrm{PeWe}$ ). The graphs are random graphs $G_{n, p}$ with parameters $n=100,300,500,1000$ and $p=0.4,0.5,0.6$.

that the ants algorithm is any better than simple (memoryless) repetition of, say RLF.

Unfortunately, such unjustified conclusions can also be found elsewhere, even in [11]. Here we first compare two versions of ANTCOL with repeated RLF. The experiments show that the version of ants algorithm which uses RLF is on average better than simple repetition of RLF, while the repeated RLF algorithm beats the version of ANTCOL using DSATUR subroutine. Then we compare the ants algorithm with the Petford-Welsh algorithm. The latter is in our experiments substantially better than the ANTCOL algorithm.

Although there is a library of benchmark problems for graph coloring available on the world wide web [10], in this experiment we decided to use the instances of random graphs of the same type as in [6] in order to be consistent with our main reference.

The idea of our experiment is to make a "fair" comparison of the ANTCOL algorithm with the RLF and Petford-Welsh algorithms. We do a rough analysis of algorithm running times in terms of changes of colors of single vertices in order to give comparable running times for all the algorithms. Since ANTCOL algorithms call RLF as a subroutine and it is easy to count the number of calls of RLF, we compare a run of ANTCOL with this number of repetitions of RLF. Furthermore, an iteration of the PetfordWelsh algorithm is a change of a color of one vertex and one run of RLF is hence comparable with $n$ (the number of vertices of the graph) iterations of the Petford-Welsh algorithm. The results are summarized in two tables.

The rest of the paper is organized as follows. First, we briefly recall the graph coloring problem and generation of random graphs. Then we give details of the algorithms.

\section{Definitions}

If $G$ is a graph, we write $V(G)$ for its vertex set and $E(G)$ for its edge set. $E(G)$ is a set of unordered pairs $x y=\{x, y\}$ of distinct vertices of $G$.

A proper $k$-coloring of vertices of a graph $G$ is a function $f$ from $V(G)$ onto a set (of colors) $X,|X|=k$, such that $u v \in E(G)$ implies $f(u) \neq f(v)$. The smallest number $k$ for which a proper $k$-coloring exists is the chromatic number of $G, \chi(G)$. An optimal coloring is one which uses exactly $\chi(G)$ colors. A set $S \subseteq V(G)$ is independent if $x y \notin E(G)$ for any pair of vertices $x, y \in S$.

A random graph $G_{n, p}$ is generated as follows. For any pair of vertices $u$ and $v$ the edge $u v$ is inserted with probability $p$, independently. This is one of the usual random graph models [2]. 


\begin{tabular}{|r|r||r||l|l||l|l|l|}
\hline \multicolumn{1}{|c||}{} & \multicolumn{1}{c||}{ RLF } & \multicolumn{3}{c||}{ ANTCOL } & \multicolumn{3}{c|}{ Petford-Welsh } \\
$n$ & $p$ & & RLF & DSATUR & $T=0.2$ & $T=0.3$ & $T=0.4$ \\
\hline \multirow{3}{*}{100} & 0.4 & 13.00 & 12.70 & 13.95 & 12.30 & 12.00 & 12.00 \\
& 0.5 & 16.05 & 15.20 & 16.10 & 15.05 & 14.85 & 14.95 \\
& 0.6 & 19.65 & 18.65 & 18.75 & 18.15 & 17.90 & 18.35 \\
\hline \multirow{3}{*}{300} & 0.4 & 30.15 & 28.90 & 30.40 & 27.90 & 26.70 & 27.95 \\
& 0.5 & 38.20 & 35.70 & 38.10 & 34.60 & 33.35 & 36.10 \\
& 0.6 & 47.35 & 43.70 & 47.30 & 42.25 & 41.15 & 45.30 \\
\hline \multirow{3}{*}{500} & 0.4 & 46.00 & 45.80 & 54.00 & 41.75 & 40.00 & 41.75 \\
& 0.5 & 58.30 & 55.60 & 67.80 & 52.40 & 50.45 & 56.60 \\
& 0.6 & 72.95 & 68.20 & 83.80 & 64.30 & 63.20 & 71.25 \\
\hline
\end{tabular}

Table 2. Comparison of long runs (nants $=300,15000 \mathrm{RLF}, 15000 n \mathrm{PeWe}$ ). The graphs are random graphs $G_{n, p}$ with parameters $n=100,300,500$ and $p=0.4,0.5,0.6$.

\section{ANTCOL[6]: Ants Algorithm for Graph Coloring}

The algorithm is based on observations of an ant colony in search of a nearby feeding source. Entomological studies have shown that an ant colony converges towards an optimal solution whereas each single ant is unable to solve the problem by itself within a reasonable amount of time.

Colorni et al [4] first developed Ant System for tackling the so-called Assignment Type Problems (ATPs). Costa and Hertz [6] adapted the principles of Ant System to solve the graph coloring problem (which is an instance of ATPs). The heart of their algorithm is a loop with some (they suggested up to 50) iterations. The algorithm builds a feasible solution at each iteration. The solution is produced by a colony of ants. The colonies of nants $=100$ and 300 ants are used in their experiments. The role of each ant is to color the graph in a constructive way. Initially, the ants do not have any knowledge of the problem to be solved. At the end of each iteration the experience of every ant is memorized in an $n \times n$ matrix $M$ (where $n=V(G)$ ). The value of an entry $M_{u v}$ in $M$ is proportional to the quality of the colorings obtained by giving the same color to $u$ and $v$. At each step of a constructive method the next vertex is chosen with probability which depends on the values in $M$. For details, the reader is referred to [6].

The authors used randomly generated graphs to test the efficiency of the algorithm. The experiments presented in [6] show that the tuned ANTCOL algorithm using RLF as a constructive method performs better than ANTCOL us- ing DSATUR as a constructive method. The results on random graphs $G_{n, p}$ for $n=100,300,500$, and 1000 and for $p=0.4,0.5$ and 0.6 with both versions of ANTCOL in Table 1 and Table 2 are taken from [6].

\section{Constructive Methods: Greedy, DSATUR and RLF}

Constructive methods for graph coloring are often implemented to quickly produce an upper bound of the chromatic number.

Many constructive methods for graph coloring have been proposed. Most of them color each vertex in turn with the smallest possible color. The quality of the resulting coloring strongly depends on the order in which vertices are examined. The ordering of vertices can be either static or dynamic.

An example of static ordering is to preorder vertices according to their degree. This is known as the Welsh-Powell algorithm [18]. Another example of static ordering is a random (pre)ordering of vertices. If the ordering is random, we call the constructive algorithm the greedy coloring heuristics.

The dynamic ordering is generally more effective since the choice of the next vertex to be colored is based on the previous color assignments. The most important methods using dynamic ordering are DSATUR [1] and RLF [9]. The experiments presented in [6] show that better coloring can be obtained using RLF. In our experiment we therefore used only RLF, which is explained in more detail below. 
The RLF algorithm builds the classes of colors sequentially. The method selects vertices one color class at a time. Assume $V_{i}$ is the next color class to be completed. Let $W$ be the set of uncolored vertices which can be included in the independent set $V_{i}$ and let $B$ be the set of uncolored vertices which can no longer be included in the independent set because they are adjacent to at least one vertex in $V_{i}$. The algorithm builds $V_{i}$ as follows:

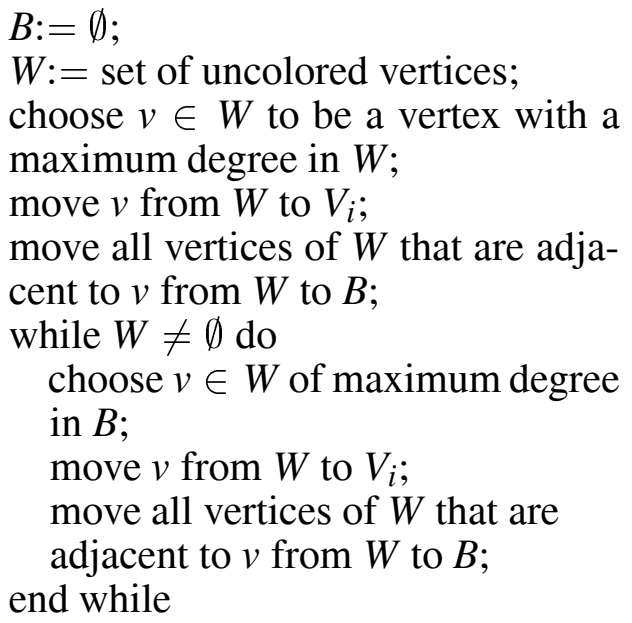

The strategy of RLF is to make $\left|V_{i}\right|$ large and the graph induced by the remaining vertices sparse.

To compare the ants algorithm and RLF with the same assumptions a comparable repeated RLF algorithm using solely RLF is used. Our RLF algorithm is a simple repetition of 5000 (15000) independent runs of RLF, and only the best result obtained so far is stored in the memory. This corresponds to 50 iterations of ANTCOL with 100 (300) ants. However, note that the ANTCOL needs both more time and space since it stores and updates the matrix $M$.

We found that RLF was on average worse than ANTCOL using subroutine RLF. However, it is interesting that simple repetitions of RLF were on average better than ANTCOL using DSATUR.

We also did a few experiments with repeated greedy coloring heuristics. Not surprisingly, it appeared to be much worse than repeated RLF.

\section{Petford-Welsh Type Algorithms, PeWe}

The algorithm we use is a generalization of the algorithm of Petford and Welsh [12]. This algorithm and some of its generalizations have proved to be reasonably good heuristics for coloring various types of graphs including random $k$-colorable graphs, DIMACS challenge graphs [10], frequency assignment "realistic" graphs, and others $[12,19,20,14,3,17]$.

In [3] we used the algorithm as given in [19]. Here, instead of starting with a random 2-coloring, we start with a coloring obtained by the greedy coloring algorithm. The order of vertices colored by greedy algorithm is random. The main loop is the same as in $[19,3]$. We now briefly recall the algorithm for completeness of the presentation.

$$
\begin{aligned}
& \text { get a random order of vertices; } \\
& \text { run a greedy coloring algorithm; } \\
& \text { while not stopping condition do } \\
& \text { if the coloring is proper then recolor } \\
& \text { vertices of the maximal color } \\
& \text { select a bad vertex } v \text { (randomly) } \\
& \text { assign a new color to } v \\
& \text { end while }
\end{aligned}
$$

The greedy coloring always takes the minimal color which does not violate any constraints.

Eventually the algorithm finds a proper coloring with $k$ colors for some $k$. We hence know that $\chi(G) \leq k$. The role of the first line in the while loop is to reduce the number of colors in the case when a proper coloring was found. All vertices of color $k$ are recolored to obtain a (usually not proper) coloring with at most $k-1$ colors. The algorithm continues and tries to find a proper coloring with fewer than $k$ colors.

In the second line of the while loop, a bad vertex is selected uniformly at random among the bad vertices, i.e. vertices which are endpoints of some edge which violates a constraint.

A new color is assigned at random. The new color is taken from the set $\{1 \ldots k\}$ by sampling according to distribution defined as follows: The probability of color $i$ to be chosen as a new color of vertex $v$ is proportional to

$$
\exp \left(S_{i} / T\right)
$$

where $S_{i}$ is the number of edges with one endpoint at $v$ and violating the constraint provided color of vertex $v$ is given color $i$. 
$T$ is a parameter of algorithm, which may be called temperature because of the analogy to temperature of the simulated annealing algorithm.

The stopping criteria we choose is a time limit (in terms of the number of calls to the function which computes a new color) and the number of colors with which we want to color the graph. The algorithm stops if either of conditions is satisfied. If we do not want to guess the chromatic number, we can simply take 1 or 2 for the desired number of colors. This can never be achieved, so the only stopping condition is the time limit. Since it starts with a greedy coloring, we always have a proper assignment (with a number of colors which may be bigger than optimal). This assignment can be regarded as an approximate solution.

Remark: there are two decisions which are of crucial importance for the behaviour of the algorithm: to define the number of iterations before restart (or define a stopping criteria) and to define a good value for parameter $T$. We have no complete answer to these two questions. The choices we made work well on instances tested, but in general fine tuning is probably needed as is the usual case with other heuristics of similar type such as simulated annealing, tabu search, genetic algorithms, etc. The idea of adapting the temperature is addressed in [15].

In order to be comparable with RLF and ANTCOL, we run Petford-Welsh algorithm with $5000 n$ iterations and $15000 n$ iterations. Each iteration of Petford-Welsh algorithm is a change of color of one vertex. Since a RLF changes all $n$ vertices, one RLF corresponds to approximately $n$ iterations of Petford-Welsh algorithm. The running times are in range from less than a minute for runs of $500 n$ iterations on small graphs $(n=100)$ up to 88 minutes CPU for $500 n$ iterations on large graphs $(n=1000)$ (on AlphaServer 1000 4/200 VAX/VMS).

We took three values of the parameter $T: 0.2$, 0.3 and 0.4. These values were guessed, based on our experience with the algorithm on other classes of graphs. More tuning could only improve the performance of the algorithm.

Remark: According to Costa and Hertz, the best average result on $G_{100,0.5}$ is 14.95 , obtained in [7] and in [5]. The Petford-Welsh algorithm scored 14.95 with both $T=0.3$ and $T=0.4$ in short version. The longer version results were: 14.80 at $T=0.3$ and 14.90 at $T=0.4$.

\section{Conclusion}

We conclude by restating the main message of this paper. Indeed, a cooperating colony of ants can color graphs better than many independent ants. However, the ants algorithm is not competitive with some other graph coloring algorithms known from the literature, at least on the set of instances tested here and in [6]. In particular,

- ANTCOL using RLF gives better results than a simple repetition of RLF in most cases. Surprisingly, the repetead RLF is comparable with ANTCOL on large graphs and even more efficient for $G_{500,0.4}$, $G_{1000,0.4}, G_{1000,0.5}$ (see Table 1).

- the quality of the results of the PetfordWelsh algorithm is very high when compared with the other algorithms considered in the paper. Note that better or comparable solutions to ANTCOL are produced on wide range of parameter $T$.

\section{Acknowledgement}

The authors wish to thank John Shawe-Taylor for careful reading of the final version of the paper.

\section{References}

[1] D. BRELAZ, New methods to color vertices of a graph, Communications of the ACM 22 (1979) 251-256.

[2] B. Bollobas, Random graphs, Academic Press, London 1985.

[3] B. Chamaret, S. Ubeda, J. Žerovnik, A Randomized Algorithm for Graph Coloring Applied to Channel Allocation in Mobile Telephone Networks, in Proceedings of the 6th International Conference on Operational Research KOI'96 (T.HUNJAK, LJ.MARTIĆ, L.NERALIĆ, Eds.), (1996) pp.25-30, Hrvatsko društvo za operacijska istraživanja, Zagreb. 
[4] A. Colorni, M. Dorigo And V. Maniezzo, Distributed optimization by ant colonies, in Proceedings of the first European Conference on Artificial Life (F.J. VARELA AND P. BOURGINE, Eds.), (1991) pp.134-142, MIT Press/Bradford Books, Cambridge, Massachusetts.

[5] D. Costa, A. Hertz And O. Dubuis, Embedding of a sequential algorithm within an evolutionary algorithm for coloring problems in graphs, Journal of Heuristics, 1 (1995), 105-128.

[6] D. Costa AND A. Hertz, Ants can colour graphs, Journal of the Operational Research Society, $\mathbf{4 8}$ (1997), 295-305.

[7] C. Fleurent AND J.A. Ferland, Genetic and hybrid algorithms for graph coloring, Annals of Operations Research, 63 (1996), 437-461.

[8] M.R. GAREY, D.S. JohnSON, Computers and Intractability, Freeman, San Francisco 1979.

[9] F. LEIGHTON, A graph coloring algorithm for large scheduling problems, J. Res. National Bureau of Standards, 84 (1979), 489-505.

[10] D.S. Johnson, M. TRICK,(editors) Cliques, Coloring and Satisfiability, DIMACS Series in Discrete Mathematics and Theoretical Computer Science, vol.26, AMS, Rhode Island 1996. (DIMACS coloring benchmarks available via ftp at rutgers.dimacs.edu)

[11] D.S. Johnson, C.A. Aragon, L.A. McGeOCH, C. SCHEVON, Optimization by Simulated Annealing: an Experimental Evaluation - Part II (Graph Coloring and Number Partitioning), Operations Research 31 (1991), 378-406.

[12] A.D. PeTFORD, D.J.A. Welsh, A randomized 3colouring algorithm, Discrete Mathematics $\mathbf{7 4}$ (1989), 253-261.

[13] J. Shawe-TaYlor, J. ŽErovniK, Boltzmann Machines with Finite Alphabet, in Artificial Neural Networks 2, vol.1. (I.ALEKSANDER, J.TAYLOR Eds.), (1992) pp.391-394, Elsevier Science Publishers B.V., Amsterdam.

[14] J. Shawe-TaYlor, J. ŽErovniK, Analysis of the Mean Field Annealing Algorithm for Graph Colouring, Journal of Artificial Neural Networks, 2 (1995), 329-340.

[15] J. SHAWE-TAYloR, J. ŽEROVNIK, Adapting temperature for some randomized local search algorithms, Preprint series Dept. Math., University of Ljubljana, vol.36 (1998) no.614.

[16] K. STECKE, Design Planning, Scheduling and Control Problems of Flexible Manufacturing, Annals of Operations Research, 3 (1985), 3-12.

[17] S. UBÉDA, J. ŽEROVNIK, A randomized algorithm for graph coloring applied to channel assignment problem, in Proceedings of the 4th International Symposium on Operational Research (V.RUPNIK, L.ZADNIK STIRN, S.DROBNE, Eds.), (1997) pp.361366, Slovenian Society INFORMATIKA - Section for Operational Research, Ljubljana.
[18] D.J.A. Welsh, M.B. Powell, An upper bound for the chromatic number of a graph and its application to timetabling problems, The Computer Journal, 10 (1967), 85-86.

[19] J. ŽEROVNIK, A randomised heuristical algorithm for estimating the chromatic number of a graph, Information Processing Letters, 33 (1989), 213-219.

[20] J. ŽEROVNIK, A Randomized Algorithm for $k$ colorability, Discrete Mathematics, 131 (1994), 379-393.

Received: March, 1999

Revised: January, 2000

Accepted: January, 2000

Contact address:

Aleksander Vesel

Department of Mathematics, PEF

University of Maribor

Koroška c. 160

SI-2000 Maribor Slovenia

e-mail: vesel@uni-mb.si

Janez Žerovnik

Faculty of Mechanical Engineering

University of Maribor Smetanova 17

SI-2000 Maribor Slovenia and

Department of Theoretical Computer Science

IMFM, Jadranska 19 SI-1111 Ljubljana Slovenia

e-mail: janez.zerovnik@imfm.uni-lj.si.

JANEZ ZEROVNIK is associate professor of mathematics at University of Maribor and has a part time position at Institute of Mathematics, Physics and Mechanics in Ljubljana. He received Ph.D. in computer science from University of Ljubljana in 1992 and Ph.D. in mathematics from Technical University of Graz, Austria. His research interests include graph theory, combinatorial optimization, and applications.

ALEKSANDER VESEL is assistant professor of computer science at University of Maribor and has a part time position at Institute of Mathematics, Physics and Mechanics in Ljubljana. He received Ph.D. in computer science from University of Maribor in 1996. His current research interests are algorithms for graph problems, combinatorial optimization, and applications. 